\title{
Effect of antenatal corticosteroids in late preterm delivery on neonatal morbidity: A Randomised Controlled Trial
}

\author{
Anisah Yahya ${ }^{1}$, Hajaratu Sulayman ${ }^{1}$, Isa Abdulkadir ${ }^{1}$, and Lawal Bola ${ }^{2}$ \\ ${ }^{1}$ Ahmadu Bello University Teaching Hospital \\ ${ }^{2}$ Ahmadu Bello University
}

January 19, 2022

\begin{abstract}
Objective: The use of antenatal corticosteroids beyond 34 weeks of gestation remains a debate. This study sought to determine the effect of use of antenatal corticosteroids in late preterm delivery on neonatal morbidity. Design: It was a randomized double-blind placebo and active controlled multi arm trial. There were two study groups and one control group. Setting: It was conducted at the department of Obstetrics and Gynaecology and the Department of Paediatrics of Ahmadu Bello University Teaching Hospital Zaria. Population: Pregnant women at 34 weeks 0 days to 36 weeks 6 days of gestation scheduled for elective/emergency delivery were recruited for the study. Methods: The study groups had dexamethasone and betamethasone respectively while the control group had a placebo. Main outcome: The primary outcome was incidence of respiratory distress syndrome. Results: A total of 138 mothers and 146 preterm neonates were included in the study with 48 exposed to placebo, 49 exposed to betamethasone and 49 exposed to dexamethasone. A pairwise analysis was done to test for difference between the groups. There was no statistically significant difference in the incidence of respiratory distress syndrome between the dexamethasone vs placebo $(\mathrm{p}=0.98$, RR 1 , CI 0.06-16.89), betamethasone vs placebo $(\mathrm{p}=0.98 \mathrm{RR} 1$, CI $0.09-11.55)$ and dexamethasone vs betamethasone ( $\mathrm{p}=0.32$, RR 0.5, CI 0.09-2.42) Conclusion: Antenatal corticosteroids may decrease the need for neonatal resuscitation at birth in late preterm neonates.
\end{abstract}

\section{TITLE PAGE}

Title: Effect of antenatal corticosteroids in late preterm delivery on neonatal morbidity: A Randomised Controlled Trial

Authors

1. Anisah Yahya. Department of Obstetrics and Gynecology, College of Medical Sciences, Ahmadu Bello University/Ahmadu Bello University Teaching Hospital, Zaria, Nigeria.

2. Hajaratu Umar Sulayman. Department of Obstetrics and Gynecology, College of Medical Sciences, Ahmadu Bello University/Ahmadu Bello University Teaching Hospital, Zaria, Nigeria.

3. Isa Abdulkadir. Department of Paediatrics, College of Medical Sciences, Ahmadu Bello University/Ahmadu Bello University Teaching Hospital, Zaria, Nigeria.

4. Bola Biliaminu Lawal. Department of Community Medicine, College of Medical Sciences, Ahmadu Bello University Zaria, Nigeria.

Corresponding author

Anisah Yahya. Department of Obstetrics and Gynecology, College of Medical Sciences, Ahmadu Bello University/Ahmadu Bello University Teaching Hospital, Zaria, Nigeria

+2348035925175, anisahy@yahoo.com, anisahungogo@gmail.com ayahya@abu.edu.ng 
Running title: Antenatal corticosteroids in late preterm delivery

\section{ABSTRACT}

Objective: The use of antenatal corticosteroids beyond 34 weeks of gestation remains a debate. This study sought to determine the effect of use of antenatal corticosteroids in late preterm delivery on neonatal morbidity.

Design: It was a randomized double-blind placebo and active controlled multi arm trial. There were two study groups and one control group.

Setting: It was conducted at the department of Obstetrics and Gynaecology and the Department of Paediatrics of Ahmadu Bello University Teaching Hospital Zaria.

Population: Pregnant women at 34 weeks 0 days to 36 weeks 6 days of gestation scheduled for elective/emergency delivery were recruited for the study.

Methods: The study groups had dexamethasone and betamethasone respectively while the control group had a placebo.

Main outcome: The primary outcome was incidence of respiratory distress syndrome.

Results: A total of 138 mothers and 146 preterm neonates were included in the study with 48 exposed to placebo, 49 exposed to betamethasone and 49 exposed to dexamethasone. A pairwise analysis was done to test for difference between the groups. There was no statistically significant difference in the incidence of respiratory distress syndrome between the dexamethasone vs placebo ( $\mathrm{p}=0.98, \mathrm{RR} 1$, CI 0.06-16.89), betamethasone vs placebo $(\mathrm{p}=0.98 \mathrm{RR} 1$, CI $0.09-11.55)$ and dexamethasone vs betamethasone $(\mathrm{p}=0.32$, RR 0.5, CI 0.09-2.42)

Conclusion: Antenatal corticosteroids may decrease the need for neonatal resuscitation at birth in late preterm neonates.

Funding: The research was funded by Tertiary Education Trust Fund (TETFUND) of Nigeria.

Trial registration: ClinicalTrial.gov, NCT03446937

Keywords: Antenatal corticosteroids, late preterm delivery, neonatal morbidity.

\section{INTRODUCTION}

Preterm birth is a significant cause of neonatal morbidity and mortality. The use of antenatal corticosteroids before 34 weeks of gestation has been proven to prevent respiratory distress syndrome and other morbidities in neonates.(1) However, limited evidence is available for its use in the late preterm period.(2),(3)

Respiratory distress syndrome is one of the most important causes of neonatal morbidity and mortality in preterm births. Other causes of neonatal morbidity and mortality in preterm neonates include intraventricular haemorrhage, sepsis, periventricular leucomalacia and necrotizing enterocolitis. In preterm neonates, surfactant insufficiency, immaturity and poor lung development result in respiratory distress in preterm infants. As gestation advances, chances of survival improve due to increasing maturity of the respiratory system. Black neonates are said to have lower rates of respiratory distress syndrome compared to non-black neonates.(4) This has been attributed to qualitative difference in the surfactants and possible anatomical difference in fetal lung development.(4) Despite this, there is some evidence that infant mortality due to respiratory distress may actually be affected by genetics race and ethnicity.(5)

Even though adequate fetal lung maturation for survival is expected to be achieved by 34 weeks of gestation, neonatal and childhood complications are still common in preterm neonates delivered after 34 weeks of gestation when compared to those delivered at term. (5),(6), (7). This led to the recommendation by National Institute Of Child Health And Human Development during a workshop in 2005 to direct research 
to evaluate infants who are born between 34 and 36 weeks gestation especially with regards to the benefits of use of antenatal corticosteroids in the population. (8)

This study sought to determine the effect of antenatal corticosteroids on neonatal respiratory distress syndrome and other neonatal complications in women at risk of late preterm delivery in our clinical setting.

\section{METHODS}

\section{Trial Design}

The study was a randomized double-blind placebo and active controlled multi arm trial. Pregnant women at risk of late preterm delivery or scheduled for delivery in the late preterm period were categorized into three groups. There were two study groups and one control group.

\section{Participants}

Pregnant women at 34 weeks 0 days to 36 weeks 6 days of gestation and a probability of delivery in the late preterm period irrespective of diagnosis were recruited for the study. Also, pregnant women scheduled for elective/emergency delivery in the late preterm period irrespective of indication and route of delivery were recruited.

The study was conducted between February 2018 and May 2019 at the department of Obstetrics and Gynecology and department of Paediatrics Ahmadu Bello University Teaching Hospital, Shika. Recruitment was stopped when desired sample size was achieved.

Gestational age was calculated from the date of last menstrual period or early scan (before 20

weeks of gestation).

Probability of delivery is defined as:

Uterine contractions and cervical effacement of [?] $80 \%$

Preterm labor: Uterine contractions and cervical dilatation of $3 \mathrm{~cm}$.

Premature rupture of membranes: Evidence of liquor drainage by the bedside.

\section{Intervention}

The first study group received antenatal corticosteroids in the form of intramuscular dexamethasone sodium phosphate (produced by Taizhou Overseas International Ltd. 126-128 Qingnian Road Jiaojiang, Taizhou,

Zhejiang, China).

The second study group received antenatal corticosteroids in the form of intramuscular betamethasone sodium phosphate (USP 12601, CAT No 1068004, Lot: R004E0, Twin brook Pkwy, Rockville MD, +1-301881-0666, Singapore)

The control group received intramuscular water for injection as placebo (Produced by Juhel pharmaceutical limited, Nigeria).

\section{Outcome measures}

Primary outcome measure:

\section{Respiratory Distress:}

(a)Tachypnoea with grunting, recession, or nasal flaring with diffuse reticulogranular infiltrate on X-ray.

(b) Respiratory distress requiring the need for respiratory support by 72 hours of age and will consist of one or more of the following:

Requirement for intranasal oxygen for [?] 4 hours 
Requirement for Bubble Continuous Positive Airway Pressure.

Secondary outcome measures:

1. Transient tachypnoea of the new born: Respiratory distress resolved within 72 hours of life without diffuse reticulogranular infiltrate.

2. Admission into Special Care Baby Unit (SCBU)/Neonatal Intensive Care Unit (NICU):

Stay in the SCBU/NICU for more than 12 hours within the first 72 hours of life excluding admission after discharge. Period of observation is not considered as admission. (All preterm neonates are routinely taken to the SCBU for observation immediately after delivery.)

3. Apnoea: an unexplained episode of cessation of breathing for 20 seconds or longer, or a shorter respiratory pause associated with bradycardia, cyanosis, pallor, and/or marked hypotonia.

4. Need for resuscitation at birth: Failure of the new born to cry and/or initiate spontaneous breathing at birth. To be determined by skilled birth attendants that conducts the delivery.

5. Neonatal hypoglycaemia: Blood glucose level less than $2.2 \mathrm{mmol} / \mathrm{L}$ at 3 hours after delivery.

6. Neonatal sepsis: Hypothermia or pyrexia and clinical evidence of sepsis in any organ/system.

7. Neonatal death.

8. Maternal sepsis: Clinical evidence of endometritis characterized by pyrexia [?] 38.0 sub-involution of uterus and foul-smelling lochia.

\section{Sample size}

The sample size for each group was determined by using the statistical formula for comparison of proportions as follows:

$\mathrm{n}=1 /((1-\mathrm{f})) \times\left(2 \times \llbracket\left(\mathrm{Z} \_\alpha+\mathrm{Z} \_\beta\right) \rrbracket 2 \times \mathrm{P} \times(1-\mathrm{P})\right) / \llbracket\left(\mathrm{P} \_0-\mathrm{P} \_1\right)^{2}$

Where $\mathrm{n}=$ minimum sample size

Po $=$ the proportion of participants in the control group that was expected to develop respiratory morbidity. In a study by Porto et al $23 \%$ (0.23) was reported.

$\mathrm{P} 1=$ the proportion of participants in the experimental group that was expected to exhibit the outcome of interest which is respiratory morbidity. A reduction to $10 \%(0.10)$ will be considered clinically significant in this study.

$\mathrm{Z} \alpha=$ was determined from a statistical table based on the value of the level of significance. For this study, it was set at 0.05 . Therefore, $\mathrm{Z} \alpha=1.96$.

$\mathrm{Z} \beta=$ was determined from a statistical table based on the acceptable power of comparison between 2 groups. For this study, power of $80 \%$ (0.80) was used, therefore $\mathrm{Z} \beta=0.84$.

$\mathrm{f}=$ was the proportion of study participants who are expected to be lost to follow up. For this study $\mathrm{f}=10 \%(0.1)$

$\mathrm{n}=49.1$

Each group was rounded up to 50 . 


\section{Randomization}

The WINPEPI version 11.65 software (75) was used to generate the table of random numbers.

The table of random numbers was developed and kept with the research unit of the pharmacy

department, Ahmadu Bello University Teaching Hospital, where the study drugs were kept and dispensed for use once patients were recruited for the study. The randomization code was only made available to the principal investigator after completion of the trial for data analysis.

Patients that fulfilled the inclusion criteria were enrolled by the team on call or managing team as they presented to the hospital. After obtaining a written consent, the participants were requested to pick one of the brown envelopes which contained the randomization number. A

proforma was then filled for the participants indicating the randomization number. The randomization number was taken to the pharmacy unit by the attending staff where the drugs were dispensed for administration to the participants.

\section{Blinding}

All drugs were constituted and dispensed by a research assistant from the research unit of the

pharmacy department of Ahmadu Bello University Teaching Hospital in sterile syringes for immediate administration. All drugs were exactly the same in appearance and volume. The principal investigator, research assistants and special care baby unit/neonatal intensive care unit staff were not aware of the group the women and neonates belonged to because all drugs appeared exactly the same at point of administration.

\section{Statistical methods}

The data obtained was analysed using SPSS version 23.0. A pairwise analysis (9) was done

to determine where the differences exist between the groups. Pairwise analysis (betamethasone VS placebo, dexamethasone vs placebo, betamethasone vs dexamethasone) was done for statistical analysis. The sociodemographic characteristics, reproductive profile, and perinatal characteristics of the women and their neonates in the group were compared with students t-test for continuous variables and Pearson's Chi Square $\left(\chi^{2}\right)$ test and Fisher's test for discrete variables with normal distribution and Mann-Whitney U test for continuous variables or those with non-normal distribution. $\mathrm{P}$ values for all tests were two tailed and the level of significance was defined at $5 \%$. Relative risk with confidence interval was used to determine the effects of corticosteroid treatment on perinatal and maternal outcomes. Maternal and perinatal characteristics of the participants that were statistically significant between the groups were subjected to logistic regression to determine their effect on the statistically significant outcome measures.

\section{Funding}

The study was funded by the tertiary education trust fund (TETFUND) of the federal republic of Nigeria. However, the funding body did not participate in the design, implementation and write up of the study.

\section{RESULT}

\section{Recruitment}

The study was conducted between February 2018 and May 2019. Recruitment was stopped

when desired sample size was achieved.

During the period of study, a total of 150 pregnant women were randomized for the study. Out of these women, 5 women carried their pregnancies to term, 4 withdrew consent, 2 were discharged home and subsequently lost to follow up and 1 had intrauterine fetal death. The remaining 138 pregnant women were included in the study. Among these women, there were 6 sets of twins and a set of triplets. This gave a 
total number of 146 neonates in the study. Of these neonates, 48 were exposed to antenatal placebo. 49 were exposed to antenatal betamethasone and 49 were exposed to antenatal dexamethasone.

\section{DISCUSSION}

\section{MAIN FINDINGS}

The incidence of respiratory distress syndrome among late preterm neonates in the placebo group, betamethasone group and dexamethasone group were $2.1 \%, 0 \%$ and $2 \%$ respectively. The incidence of respiratory distress varies with race.(4) Low incidence of respiratory distress syndrome have been reported among blacks.(4) and may explain the low incidence of respiratory distress in our study coupled with the fact that compared to other categories of preterm births, its incidence is lower in late preterm neonates. There was no significant difference between the group exposed to placebo versus antenatal betamethasone $(\mathrm{p}=0.98$, RR 1.0, CI 0.09-11.55), the group exposed to placebo versus antenatal dexamethasone $(\mathrm{p}=0.98, \mathrm{RR}=1.0$, CI 0.06-16.87) and the group exposed to antenatal betamethasone versus antenatal dexamethasone $(\mathrm{p}=0.31$, $\mathrm{RR}=0.5$, CI 0.09-2.42). This is similar to the findings of Porto et al(10) where he reported low incidence of respiratory distress among late preterm neonates exposed to antenatal betamethasone and those exposed to placebo ( $1.4 \%$ and $0.8 \%$ respectively) with no significant difference in the incidence of respiratory distress between the 2 groups $(\mathrm{p}=0.54$, RR 1.90). However, this is not consistent with the findings of Balci et al(11) who reported an incidence of $16 \%$ among late preterm neonates that did not receive antenatal steroids and incidence of $4 \%$ among those that received betamethasone and the difference between the two groups was statistically significant $(\mathrm{p}=0.046$, OR 0.21$)$. The fact that Balci et al(11) found a significant difference in the incidence of respiratory distress in their study may be due to the fact that they did an open label trial which may have introduced some bias unlike our study and that by Porto et al(10) which were both randomized double blinded. Though a similar double blind randomized controlled trial by Gyamfi-Bannerman et al(12) found a statistically significant higher incidence of primary outcome in the late preterm neonates in the control group, the primary outcome was a neonatal composite of treatment including the use of CPAP or high flow nasal cannula at least for 2 hours, supplemental oxygen with a fraction of inspired oxygen of at least 0.30 for at least 4 hours, extracorporeal membrane oxygen, or mechanical ventilation or stillbirth or neonatal death rather than respiratory distress alone. This may account for the difference in findings.

The incidence of transient tachypnoea of the newborn was also low in this study with one neonate (2.1\%) in the group exposed to placebo developing the outcome (Tables 2 and 3). Our finding of low incidence in transient tachypnoea was not consistent with other reports. Gyamfi-Bannerman et al(12) reported an incidence of transient tachypnoea of the newborn among late preterm neonates exposed to antenatal betamethasone $6.7 \%$ and $9.9 \%$ in those exposed placebo $(9.9 \%)$ ( $\mathrm{p}=0.002$, RR 0.68 CI $0.53-0.87$ ). A higher incidence was reported by Porto et al(10) where $24 \%$ of late preterm neonates exposed to antenatal betamethasone and $22 \%$ of those exposed to placebo developed transient tachypnoea. However, there was no significant difference between the groups. The low incidence of transient tachypnoea of the newborn in our study is in keeping with the low incidence of respiratory distress syndrome noted in the study.

This study showed a statistically significant reduction in the need for neonatal resuscitation at birth among the neonates exposed to antenatal betamethasone compared to those exposed to placebo $(\mathrm{p}<0.001, \mathrm{RR} 7.0$, CI 2.49-19.46) and also the neonates exposed to antenatal dexamethasone compared to those exposed to placebo ( $\mathrm{p}=0.010$, RR 4.0, CI 1.86-26.03). This means that neonates born to women who received placebo were 7 times more likely to need neonatal resuscitation at birth compared to neonates born to women who received betamethasone and 4 times more likely to need resuscitation at birth compared to neonates born to women who received dexamethasone. The use of betamethasone was associated with a $40 \%$ decrease in need for resuscitation at birth compared to the use of dexamethasone though this was not statistically significant $(\mathrm{p}=0.461)$.

A logistic regression analysis was done to determine the relationship between the need for resuscitation at birth and the study group of the women while adjusting for 1- and 5-minutes APGAR score that was significantly higher in the betamethasone group when compared to the placebo group. The result showed 
that after adjusting for 1- and 5-minute APGAR score there was a significant reduction in the likelihood of need for neonatal resuscitation by about $85 \%(\operatorname{ExpB}=0.157, \mathrm{p}=0.005)$ when betamethasone was used compared to placebo.

Other studies have also reported decrease in the need for neonatal resuscitation at birth in women exposed to antenatal corticosteroids when compared with women exposed to placebo.(11),(12). This finding is of significance especially in our environment where many deliveries are conducted in the absence of skilled birth attendants that can adequately offer neonatal resuscitation at birth.

The need for admission into SCBU was highest among the placebo group (12.5\%), followed by the betamethasone group $(8.2 \%)$ and least in the dexamethasone group $(4.1 \%)$. However, the differences observed were not statistically significant (Tables 2,3 and 4 ).

Apnoea was not reported in any of the study or control group. This may be because continuous electronic recording of vital signs of the neonates was not done in our study and apneic episodes are short in duration. This has been observed in other studies where a lack of correlation between nurses' records with continuous electronically recorded events have been demonstrated (13),(14). In a randomized controlled trial on the use of betamethasone for women at risk for late preterm delivery, apnoea was reported to occur in $2.3 \%$ of the group that had betamethasone and in $2.6 \%$ of the control group with no significant difference between the two groups $(p=0.57)$.(12) Other studies on the use of antenatal corticosteroids in late preterm neonates did not report on apnoea. (10),(11)

The neonates exposed to antenatal dexamethasone had the highest incidence of hypoglycemia $(14.3 \%)$, followed by those exposed to antenatal betamethasone $(8.2 \%)$. The neonates exposed to placebo had the lowest incidence of hypoglycemia $(4.2 \%)$. However, the differences were not statistically significant (Tables 2,3 and 4). Conflicting reports exist in the literature with regards to incidence of hypoglycemia in neonates following exposure to antenatal corticosteroids. While some authors(12),(15) reported increase in the rate of hypoglycemia among infants exposed to antenatal corticosteroids, others did not(6). A systematic review and meta-analysis of randomized controlled trials on the use of antenatal corticosteroids in near term and term fetuses also showed an increased risk of neonatal hypoglycemia in women who received antenatal corticosteroids at more than 34 weeks gestation.(15) However, no adverse effects were reported in all the trials. This inconsistency may be due to the fact that neonatal hypoglycemia is a common late term neonatal complication irrespective of the use of antenatal corticosteroids within the period.(16)

Incidence of neonatal and maternal sepsis were low and the differences between the groups were not statistically significant. (Tables 2, 3 and 4). This is consistent with existing literature.(12)

\section{STRENGTHS AND LIMITATIONS}

The major strength of this study is the fact that it was a double blind randomized controlled trial and both dexamethasone and betamethasone were used as antenatal corticosteroids. However, it was conducted in a single center which is a limitation.

\section{CONCLUSION}

This study found that the use of antenatal corticosteroids in late preterm delivery did not have any significant effect on incidence of respiratory distress syndrome and other neonatal morbidity but significantly reduced the need neonatal resuscitation at birth.

\section{RECOMMENDATIONS}

In view of the findings in this study we recommend the use of antenatal corticosteroids in women at risk of preterm delivery with the aim of reducing the need for neonatal resuscitation at birth.

However, there is still the need for more trials on the effect of use of antenatal corticosteroids in late preterm deliveries with the aim of preventing neonatal morbidity and minimizing side effects on both mother and neonates. 


\section{ACKNOWLEDGEMENT}

We would like to acknowledge the staff of the labour ward and the special care baby unit of Ahmadu Bello University Teaching Hospital for their support during the period of the study.

\section{DISCLOSURE OF INTEREST}

The authors declare no conflict of interest. The authors do not have any financial, personal, political, intellectual or religious affiliations/relationships with any institution or organization that may have influenced the research.

\section{CONTRIBUTION TO AUTHORSHIP}

AY was involved in the concept, design, definition of intellectual content, literature search, data acquisition, data analysis, statistical analysis, manuscript preparation, manuscript editing and manuscript review. HUS and IA were involved in the definition of intellectual content, data acquisition, manuscript editing and review. BBL was involved in data analysis, statistical analysis, manuscript editing and manuscript review.

\section{DETAILS OF ETHICAL APPROVAL}

Ethical approval to conduct the study was sought from the Health Research and Ethics Committee of Ahmadu Bello University Teaching Hospital Shika. The application was reviewed by the committee and permission to conduct the study was given on 07/08/2017 with reference number ABUTHZ/HREC/Y3/2017 (NHREC/10/12/2015; D-U-N-S-No 954524802; ABUTH/HREC/CL/05/). The initial permission to conduct the study expired on $07 / 08 / 2018$ before the desired sample size was achieved. Permission for extension of study period to $07 / 08 / 2018$ was granted after due process.

\section{FUNDING}

Funding was received from Institution Based Research funds of the Tertiary Education Trust Fund of Nigeria. The funding is part of funding for professional development and not for commercial or other purposes.

\section{REFERENCES}

1. Crowley P. Antenatal corticosteroids:A meta-analysis of randomised trial. Am J Obs Gynecol. 1995;173:322-35.

2. Roberts D, Dalziel SR. Antenatal corticosteroids for accelerating fetal lung maturation for women at risk of preterm birth. Cochrane database Syst Rev [Internet]. 2006;(3):No.: CD004454. Available from: http://www.mrw.interscience.wiley.com/cochrane/clsysrev/articles/CD004454/pdf_fs.html

3. Escobar G, Clark RH, Greene J. Short-term outcomes of infants born at 35 and 36 weeks gestation: we need to ask more questions. Semin Perinatol. 2006;30(1):28-33.

4. Berman S, Tanasijevic MJ, Alvarez JG, Ludmir J, Lieberman E, Richardson DK. Racial differences in the predictive value of the TDx fetal lung maturity assay. [Internet]. Vol. 175, American journal of obstetrics and gynecology. 1996. p. 73-7. Available from: http://www.ncbi.nlm.nih.gov/pubmed/8694078

5. Frisbie WP. The increasing disparity in infant mortality: Respiratory distress syndrome and other causes. Demography. 2004;41:773-800.

6. Liggins G, Howie R. A controlled trial of antepartum glucocorticoid treatment for the prevention of respiratory distress syndrome in premature infants. Pediatrics. 1972;50:515-25.

7. Hibbard JU. Respiratory morbidity in late preterm births. J Am Med Assoc. 2010;304(4):419-25.

8. Raju T, Higgins R. Optimising care and outcome for late preterm (near term) infants. Summary of workshop sponsored by the National Institute Of Child Health And Human Development. Pediatrics. 2006;118:1207-14. 
9. Juszczak E, Altman D, Hopewell S, Schulz K. Reporting of Multi-Arm Parallel-Group Randomized Trials Extension of the CONSORT 2010 Statement. Clinical Review. 2019;321(16):1610-20.

10. Porto A. Effectiveness of antenatal corticosteroids in decreasing respiratory disorders in late preterm infants. Randomised controlled trial. BMJ. 2011;342:d1696.

11. Balci O. The effect of antenatal corticosteroid in fetal lung maturation between 34th and 36th week of pregnancy. Gynecol Obs Invest. 2010;70:95-9.

12. Gyamfi-Bannerman C, Thom EA, Blackwell SC, Tita ATN, Reddy UM, Saade GR, et al. Antenatal Betamethasone for Women at Risk for Late Preterm Delivery. N Engl J Med [Internet]. 2016;374(14):131120. Available from: http://www.ncbi.nlm.nih.gov/pubmed/26842679

13. Brockmann P, Wiechers C, Pantalitschka T, Diebold J, Vagedes J, Poets C. Under-recognition of alarms in a neonatal intensive care unit. Arch Dis Child Fetal Neonatal Ed. 2013;98(6):F524-7.

14. Razi N, Humphreys J, Pandit P, Stahl G. Predischarge monitoring of preterm infants. Pediatr Pulmonol. 1999;27(2):113-6.

15. Saccone G, Berghella V. Antenatal corticosteroids for maturity of term or near term fetuses: systematic review and meta-analysis of randomized controlled trials. Bmj [Internet]. 2016;i5044. Available from: http://www.bmj.com/lookup/doi/10.1136/bmj.i5044

16. Harris D, Weston P, Harding J. Incidence of neonatal hypoglycemia in babies identified as at risk. J Pediatr. 2012;161(5):787-91.

\section{Hosted file}

Flowchart Figure Effect of Antenatal Corticosteroids in late preterm delivery on neonatal morbidity.do available at https://authorea.com/users/456076/articles/553240-effect-of-antenatalcorticosteroids-in-late-preterm-delivery-on-neonatal-morbidity-a-randomised-controlledtrial 